\title{
Numerical Analysis of a Highly Sensitive Surface Plasmon Resonance Sensor for SARS-CoV-2 Detection
}

\author{
Syed Mohammad Ashab Uddin ${ }^{1}$. Sayeed Shafayet Chowdhury ${ }^{2} \cdot$ Ehsan Kabir $^{3}$
}

Received: 16 October 2020 / Accepted: 3 May 2021 / Published online: 25 May 2021

(c) The Author(s), under exclusive licence to Springer Science+Business Media, LLC, part of Springer Nature 2021

\begin{abstract}
In this paper, we propose a surface plasmon resonance (SPR) structure based on Kretschmann configuration incorporating layers of silicon and $\mathrm{BaTiO}_{3}$ on top of $\mathrm{Ag}$ for real-time detection of severe acute respiratory syndrome coronavirus 2 (SARS-CoV-2) using thiol-tethered DNA as a ligand. Extensive numerical analysis based on transfer matrix theory as well as finite-difference time-domain (FDTD) technique has been performed to characterize the sensor response considering sensitivity, full width at half maxima, and minimum reflection. About 7.6 times enhanced sensitivity has been obtained using the proposed architecture for SARS-CoV-2 detection, compared to the basic Kretschmann configuration. Notably, the structure provides consistent enhancement over other competitive SPR structures for both angular and wavelength interrogations with a figure-of-merit of 692.28. Additionally, we repeated simulations for various ligate-ligand pairs to assess the range of applicability and robust performance improvement has been observed. As a result, the proposed sensor design provides a suitable configuration for highly sensitive, rapid, noninvasive biosensing which can be useful if adopted in experimental sensing protocols.
\end{abstract}

\section{Introduction}

Real-time detection of severe acute respiratory syndrome coronavirus 2 (SARS-CoV-2) with high sensitivity in a noninvasive manner is a critical research arena during this pandemic of Coronavirus disease 2019 (COVID-19) [1]. Since its emergence in December, 2019 in Wuhan, China, the devastating rapid transmission and havoc caused by this virus has resulted in the World Health Organization

Syed Mohammaad Ashab Uddin and Sayeed Shafaayet

Chowdhury have contributed equally to thiswork.

Syed Mohammad Ashab Uddin

smuddin@uci.edu

Sayeed Shafayet Chowdhury

chowdh23@purdue.edu

Ehsan Kabir

ehsan.kabir.buet@gmail.com

1 Department of EECS, University of California, Irvine 92697, USA

2 School of ECE, Purdue University, West Lafayette 47907, IN, USA

3 Department of EEE, Bangladesh University of Engineering and Technology, Dhaka 1205, Bangladesh
(WHO) classifying this outbreak as a pandemic on March 12, 2020 [2]. As per the report on Aug 6, 2020, number of confirmed cases has soared upto $18,614,177$, with a death toll of 702,642 [3]. With no proven medication or vaccines in sight still for COVID-19, significant research endeavors have focused on early diagnosis and management to control the damage [4]. The laboratory standard detection scheme is reverse transcriptase quantitative polymerase chain reaction (RT-qPCR), which attempts to identify the presence of nucleic acid-based genetic sequence of this virus [4]. However, RT-qPCR takes at least $3 \mathrm{~h}$ and requires sophisticated testing facilities [5] in addition to suffering from false positives. To cope with the rapid spread of COVID-19, highly sensitive diagnostic methods without requiring extensive lab setup is of utmost importance. Another promising diagnostic method is computed tomography (CT) scan where multiple chest X-rays are captured across different angles to obtain three-dimensional (3D) images. But the challenge using such technique lies in being able to differentiate symptoms from other lung disorders, failure in doing so results in rather low specificity of around 25\% [6] in practice. Apart from these, other methods include biochemical tests targeting the viral protein or the antibodies and contact tracing [1]. Recently, Seo et al. proposed a field-effect transistor-based sensor topology for SARS-CoV-2 detection from human 
nasopharyngeal swabs [5]. While such sensing mechanisms are being explored, there is still an urgent demand for ultrasensitive, real-time, point-of-care diagnostic methods to further aid the practitioners in fighting against COVID-19. To that end, one potential domain is the usage of biosensors [7-20]. They are preferable due to high selectivity and sensitivity, rapid response time, cost-effectiveness, and multiplexing capabilities.

In terms of optical biosensors, plasmonics is an attractive field with real-time and label-free detection features. Plasmon is the combined oscillation of the free electron in metal with respect to the restoring force of the positive nucleus. Surface plasmons (SP) are confined to the surface of the metal and they are generated at the interface of the metal having negative permittivity and a dielectric material with the interaction of electromagnetic waves such as light. When they are excited, most of the light energy is absorbed by the plasmons, and thus reflection becomes the lowest. The surface plasmon wave (SPW) propagation was first demonstrated by Otto [21]. To generate SPW, the energy or momentum of the photons of light must match with the energy or momentum of the SPs which cannot be obtained when light is incident on the metal from air as a medium. Thus, a momentum enhancing configuration is needed, and it was solved by attaching a silver metal layer to a glass substrate by Kretschmann et al. [22]. Now, this configuration is known as the basic Kretschmann configuration where light is incident on the metal layer through a glass prism, and the angle of incident is changed beyond the critical angle to excite SPW on the opposite surface of the metal. Over the years, such SPR sensor setup has undergone various modifications and been utilized in various applications. Commercially available surface plasmon resonance (SPR) and localized surface plasmon resonance (LSPR) sensors have been used for disease [23] as well as viral strains detection such as SARS, MERS, and influenza [1]. Taking into account the promise of plasmonic sensors for detection purposes, Qiu et al. [24] have developed a biosensor combining plasmonic photothermal (PPT) effect and LSPR for COVID-19 diagnosis. They utilize DNA receptors which selectively bond with RNA sequences from the SARS-CoV2 through nucleic acid hybridization. One of the key challenges of accurate detection using such plasmonic sensors is ensuring ultra-sensitive performance with excellent limit of detection. Along those lines, SPR sensing is the most mature and established technology providing very good resolution of detection. However, it is pivotal to numerically analyze the suitability of deploying such SPR sensors for detecting SARS-CoV2 and a proper sensor configuration is required to achieve desired level of performance. This motivates our study in this paper where we propose an SPR sensor configuration aiming toward SARS-CoV2 detection with high sensitivity and figure-of-merit. To enhance the overall sensor performance, we integrate layers of $\mathrm{Si}$ and $\mathrm{BaTiO}_{3}$ on top of the metal layer (Ag). Thiol-tethered DNA has been used as receptor for sensing. The proposed sensing pipeline assumes collection of samples from human nasopharyngeal swabs and passing them through the sensing channel in a liquid solution, while the sensor is illuminated from different angles. When the binding occurs between immobilized receptor on sensor surface and target biomolecules from the flowing sample, a shift in SPR angle is observed the extent of which determines the sensitivity. Our transfer matrixbased analysis corroborated with finite-difference timedomain (FDTD) simulation shows that the proposed sensor architecture is able to obtain very high sensitivity with low minimum reflection (Rmin) and improved figure-of-merit (FOM) compared to other reported results. Furthermore, we verified the robustness of the sensor design through analyzing its performance for other ligand-ligate combinations.

The rest of the paper is organized as follows: Section 2 contains the design considerations and modeling schemes for different layers, then the optimization of the sensor configuration is described in Section 3, we analyze the results in detail and perform comparative analysis with regards to other proposed sensors in Section 4 and the paper is concluded in Section 5.

\section{Design Considerations and Theoretical Modeling of Proposed Structure}

The schematic view of our proposed high sensitivity SPR biosensor configuration is shown in Fig. 1. This novel hetero structure, as shown in the figure, is composed of 5 different layers. We use BK7 glass as a coupling prism followed by a Ag layer. Ag has been proven to demonstrate better sensitivity as a substrate layer due to higher SPR ratio [25]. However, one of the major drawbacks of using $\mathrm{Ag}$ is its lesser chemical stability due to oxidation while interacting with bio molecules $[25,26]$. In our proposed design, we overcome this problem by employing 3 layers comprising of $\mathrm{Si}, \mathrm{BaTiO}_{3}$ and thiol-tethered DNA, respectively, on top of Ag. Such configuration also aids in sensitivity enhancement. In recent research, Si has shown good promise in improving sensitivity [27]. Si's ability to enhance the electric field intensity of excitation light plays major role behind this sensitivity increment [28, 29]. $\mathrm{BaTiO}_{3}$ also has desirable effects on sensitivity due to its high dielectric constant. Together with low dielectric loss, it has potential to contribute significantly to enhance sensitivity of our proposed structure [30]. Thiol-tethered DNA is used as a ligand layer for sensing medium as it has shown excellent properties as a receptor of SARS-Cov-2 [24, 31]. Having decided on the composition of various layers of our sensor design, the next step is to model them properly to simulate their response. We model each 
Fig. 1 Schematic illustration of the proposed structure

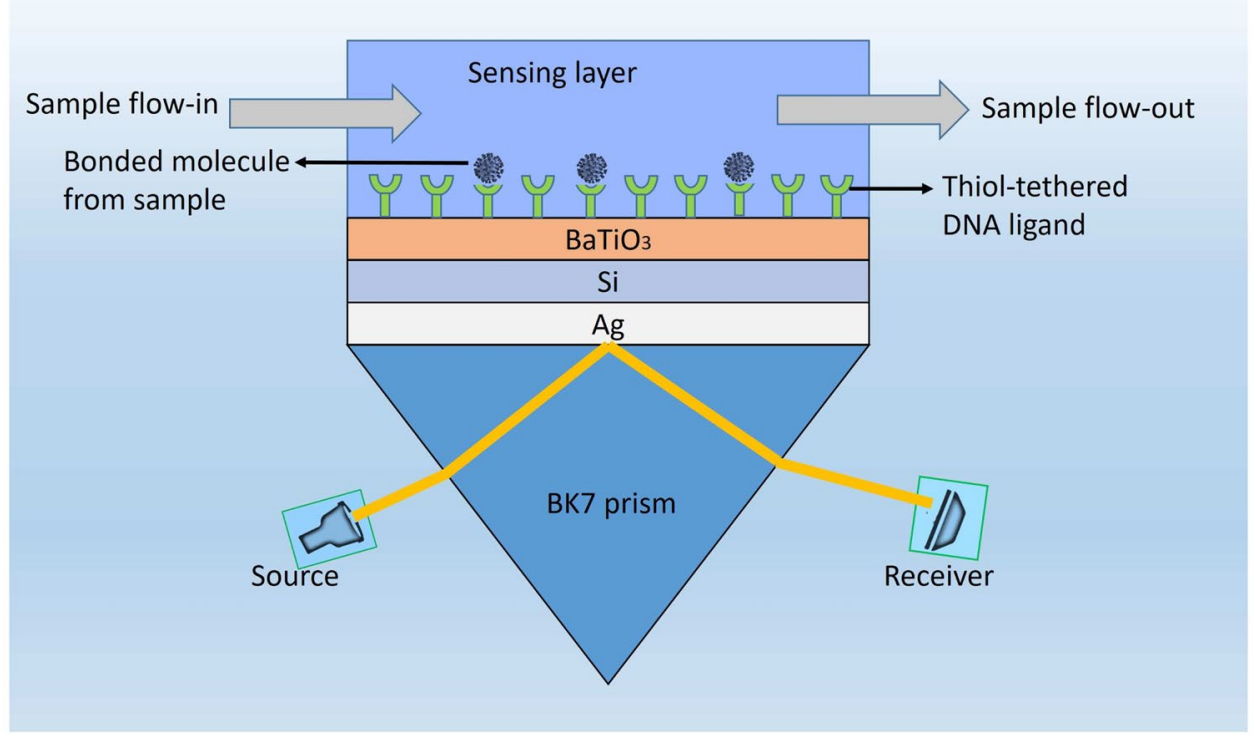

layer as a homogeneous continuous medium upto the next layer interface wherein the layer is represented by its refractive index. Hence, the next subsection focuses on modeling the refractive indices of the layers under consideration.

\section{Modeling of Layers}

The refractive indices of different layers of our structure are each calculated separately. The first layer is a coupling prism of BK7 glass. The refractive index of BK7 glass can be calculated from the following relation [32]:

$$
\begin{aligned}
n_{1}= & \left(\frac{1.03961212 \lambda^{2}}{\lambda^{2}-0.00600069867}+\frac{0.231792344 \lambda^{2}}{\lambda^{2}-0.0200179144}\right. \\
& \left.+\frac{1.01046945 \lambda^{2}}{\lambda^{2}-103.560653}+1\right)^{\frac{1}{2}}
\end{aligned}
$$

where $\lambda$ is the wavelength of incident light in micrometer. This equation is only applicable for wavelength between 0.37 to $2.5 \mathrm{um}$ [33]. The refractive index of $\mathrm{Ag}$ layer can be defined using the well-known drude model for metal [34],

$n_{m}=\left(1-\frac{\lambda^{2} \lambda_{c}}{\lambda_{p}^{2}\left(\lambda_{c}+i \lambda\right)}\right)^{\frac{1}{2}}$

where $\lambda_{p}=1.4541 \times 10^{-7} \mathrm{~m}$ and $\lambda_{c}=1.7614 \times 10^{-5} \mathrm{~m}$ are plasma and collision wavelength for Ag, respectively. The next layer of our proposed structure is silicon, the refractive index of which is defined as [29]:

$n_{S i}=A_{1}+A_{2} e^{-\frac{\lambda}{t_{0}}}+A_{3} e^{-\frac{\lambda}{t_{1}}}$

where $\lambda$ is the wavelength of the incident light in um, $\mathrm{A}_{1}=$ $3.44904, \mathrm{~A}_{2}=2271.88813, \mathrm{~A}_{3}=3.39538, t_{0}=0.058304$ and $t_{1}=0.30384$. The experimentally obtained refractive index data for $\mathrm{BaTiO}_{3}$ at our operating wavelength is 2.4043 [35]. The final layer before the sensing layer is thiol-tethered DNA. Its thickness-dependent refractive index data is obtained from experimental results [36]. It's value is 1.3-1.5 for use able thicknesses. We will see the exact value in optimization section where all the thickness will be optimized. Finally, for sensing layer modelling, SARS COV-2 can be approximated to be a solid sphere core containing RNA with radius of $r_{1}$ covered with a membrane protein of radius $r_{2}$ [37]. Hence, a volume weighted sum of the two refractive indices is calculated as the effective RI of the virus:

$n_{\text {eff }}=\frac{n_{1} V_{1}}{V_{1}+V_{2}}+\frac{n_{2} V_{2}}{V_{1}+V_{2}}=\frac{n 1+n 2\left(\eta^{3}-1\right)}{\eta^{3}}, r_{2}=\eta * r_{1}$,

where $n_{1}(V 1)$ and $n_{2}(V 2)$ are the total RI of RNA and membrane protein with corresponding volume. Since the refractive index is mainly dependent on material composition rather than geometrical size, $\eta$ value is constant for the same type of virions and for SARS COV 2, and its value is 1.25 [37]. The average refractive index of RNA is 1.54 [38] and the membrane protein refractive index varies between $1.46+/-0.006$ [39]. The average viral load is $7 * 10^{6}$ per $\mathrm{ml}$, while the maximum is $2.35 * 10^{9}$ per $\mathrm{ml}$ [40]. When sample is flown through the sensing channel, Covid 19 RNA is bonded with thiol-tethered DNA. It takes some time to build up of the surface density of virus. The standard wait time is 10 seconds. Considering the surrounding medium of the sensing chaneel is water (Refractive index is 1.33), effective refractive index of the sensing channel have been plotted against the surface density of the virus in Fig. 2. 
Fig. 2 Effective refractive index of the sensing channel vs surface density of virus

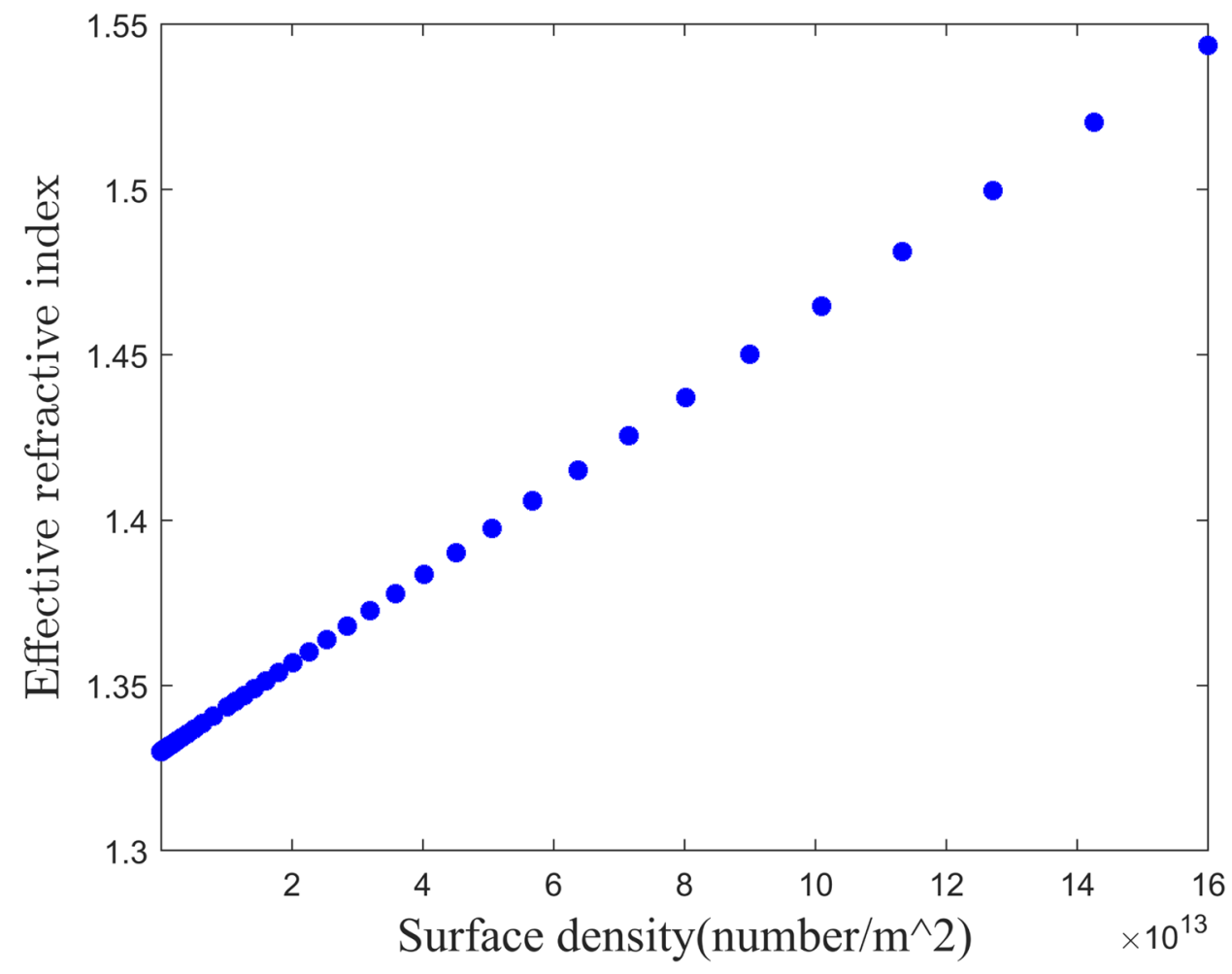

Considering the detection limit of angular shift of standard SPR devices from Biacore is 10 millidegree [41], minimum required surface density is $4 * 10^{4}$ per $\mathrm{ml}$. With the proper models of various layers, we now need to characterize the optical response of the sensor for which suitable optical excitation through appropriate irradiation is required first. This begets our next discussion on input lighting scheme.

\section{Illumination Strategy}

The choice of operating wavelength needs to be well balanced between sensitivity and optical non-linearity. Sensitivity and optical non linearity both are proportionally varied with frequency (inversely proportional to wavelength) [25]. Optical Kerr effect becomes prominent if the frequency of operation is increased such that the beam intensity is on the order of $1 \mathrm{GW} \mathrm{cm}^{-2}$ and exerts significant variations in refractive index [42]. We select operating wavelength as $633 \mathrm{~nm}$ such that sensitivity is enhanced with minimum possible Kerr effect [25, 42]. We employ ppolarized light in attenuated total internal reflection (ATR) configuration in our analysis. In this case, the incident angle must be greater than the critical angle $\theta_{c}$ of glass-silver interface, where $\theta_{c}=\frac{n_{A g}}{n_{A i r}}$. If we assume energy loss other than material absorption to be zero, then according to principle of energy conservation, the total of absorption(A), transmission (T) and reflection(R) must be equal to 1 (i.e., $\mathrm{A}+\mathrm{T}+\mathrm{R}=1$ ). Again, since transmission is zero under ATR, absorption reduces to $A=1-R$. When surface plasmons (SPs) are excited, reflection becomes minimum. As a result, this leads to the maximum transfer of light energy to the evanescent wave. With the structural and illumination setup discussed above, next it is important to formulate a theoretical framework to numerically evaluate the sensor performance, this leads to our following section on numerical modeling.

\section{Theoretical Framework}

We have performed numerical analysis to determine the change of reflectivity using transfer matrix method [43] and Fresnel equation [44] for basic Kretschmann configuration having multiple layers. Our proposed model consists of five layers with BK7 prism, silver, silicon, barium titanate, and thiol-tethered DNA being placed in parallel one after another. The thickness of each layer varies in perpendicular direction denoted as z-axis. The boundary condition at the interface of first two layers and the last two layers are considered as $Z=Z_{1}=0$ and $Z=Z_{n-1}$, respectively. The dielectric constant $\left(\epsilon_{p}\right)$ of p'th layer equals to the square of its refractive index $\left(n_{p}\right)$. These methods adopt no approximation allowing them to give accurate results rapidly. The transfer matrix expresses a relationship among the tangential components of electric and magnetic fields of the first layer and the last layer, and the relation is given as [45]-

$\left[\begin{array}{l}E_{1} \\ B_{1}\end{array}\right]=M \times\left[\begin{array}{l}E_{n-1} \\ B_{n-1}\end{array}\right]$, 
where, $E_{1}$ and $B_{1}$ are tangential components of electric and magnetic fields respectively in the interface of the first two layers, and $E_{n-1}$ and $B_{n-1}$ are the same for the interface of the $\mathrm{N}-1$ and $N^{\text {th }}$ layer. $M$ represents the characteristic matrix of the whole structure on which the reflection coefficients are dependent. For TM polarized light, it can be expressed as,

$M=\left(\prod_{k=2}^{N-1} M_{k}\right)=\left(\begin{array}{ll}M_{11} & M_{12} \\ M_{21} & M_{22}\end{array}\right)$,

where, $M_{k}=\left[\begin{array}{cc}\cos \delta_{k} & \left(\frac{-i \sin \delta_{k}}{\gamma_{k}}\right) \\ \left(-i \gamma_{k} \times \sin \delta_{k}\right) & \cos \delta_{k}\end{array}\right]$ with $\gamma_{k}=\sqrt{\frac{\mu_{k}}{\epsilon_{k}}} \times \cos \theta_{k}$ $=\frac{\sqrt{\left(\epsilon_{k}-n_{1}^{2} \sin ^{2} \theta_{1}\right)}}{\epsilon_{k}}$, and $\delta_{k}=\frac{2 \pi}{\lambda} n_{k} \cos \theta_{k}\left(Z_{k}-Z_{k-1}\right)=$ $\frac{2 \pi d_{k}}{\lambda} \sqrt{\left(\epsilon_{k}-n_{1}^{2} \sin ^{2} \theta_{1}\right)}$ in which $\mu_{k}, \epsilon_{k}, d_{k}, \theta_{1}, \lambda$ are magnetic permeability, electric permittivity, thickness of $k^{\text {th }}$ layer, incident angle, and wavelength of light, respectively.

From the above equations, we can derive that the total reflection coefficient $\left(r_{p}\right)$ as,

$r_{p}=\frac{\left(M_{11}+M_{12} \gamma_{N}\right) \gamma_{1}-\left(M_{21}+M_{22} \gamma_{N}\right)}{\left(M_{11}+M_{12} \gamma_{N}\right) \gamma_{1}+\left(M_{21}+M_{22} \gamma_{N}\right)}$,

where, $M_{11}, M_{12}, M_{21}, M_{22}$ are the components of the transfer matrix. The reflectivity $\left(R_{p}\right)$ for a TM polarized light is calculated as $\left|r_{p}\right|^{2}$.

We also utilize FDTD method to analyze our structure in order to further strengthen the validity of our investigation. For this purpose we utilize Lumerical FDTD solver. It's a commercially available and well known software for optical and photonic research. In FDTD, we consider meshing so that it can reach convergence with sufficient precision without making the simulation unnecessarily lengthy. We incorporate Conformal Variant 2' as mesh refinement and 'Auto non-uniform' as mesh type. Mesh accuracy is selected as '4'. We also override mesh in the sensitive regions as follows, $\mathrm{dx}=0.1 \mathrm{~nm}, \mathrm{dy}=0.1 \mathrm{~nm} \mathrm{dz}=0.0001 \mathrm{~nm}$. It simulates each layer with corresponding complex refractive index at the respective wavelength. We apply perfectly matched layer (PML) boundary condition along the direction of light incidence and bloch boundary condition in the direction perpendicular to it. With the structural setup and numerical framework as discussed above at hand, next we need to define some standard evaluation criteria to quantify the performance of our design which leads to our next section.

\section{Performance Parameters}

To evaluate the performance of SPR biosensor, several criteria can be utilized such as sensitivity (S), minimum reflectance (Rmin) and full width at half maximum (FWHM). These parameters can be obtained from the curve of incident angle versus reflection. To analyze the sensing performance and compare with other novel structures, we defined Figure of Merit (FOM) as follows,

$F O M=\frac{S}{F W H M \times R \min }$.

Minimum reflectance is defined as the reflectance at the surface plasmon resonance angle. To increase the detection accuracy, the value of the Rmin should be as low as possible. Resolution and signal-to-noise ratio (SNR) are dependent on FWHM. FWHM is characterized as the spectral width of SPR curve at half maxima. The most crucial parameter is the sensitivity of the sensor which is the characteristic parameter of a sensor that transforms a device into a sensor. It can be observed by monitoring the shift of the resonance angle of SPR curve due to the change in the refractive index of the sensing medium. Quantitatively, it can be calculated from the following equation,

$S=\frac{\delta \theta_{S P R}}{\delta n_{\text {res }}}$,

where $\theta_{S P R}$ is the SPR resonance angle and $n_{\text {res }}$ is the refractive index of the corresponding target molecule. While sensitivity determines the characteristic response of a sensor, the true performance of it cannot be evaluated on sensitivity solely. Therefore, for a more comprehensive analysis, we consider FOM taking into account the overall effect of S, FWHM and Rmin as mentioned before.

\section{Optimization of the Structure}

As mentioned earlier, there are five different layers in our proposed architecture, each layer has significant impact on the overall sensor performance. Therefore, to harness the maximum response out of our sensor configuration, proper optimization of design parameters needs to be performed in a synergistic manner. Here, we follow layerwise optimization procedure as outlined in [46]. First, we investigate sequentially the effects of each layer thickness on different performance parameters of the sensor. For this process, we determine the reflectivity as a function of incident angle using Eqn. (6). The minimum value of the reflectivity is termed as Rmin. It is closely related to the momentum transfer from the light beam to the surface plasmon. The better the momentum transfer, the lower the Rmin. We also calculate sensitivity as a function of incident angle utilizing Eqn. (8). It is related to the shift of the peak of resonance curve with respect to the corresponding change of the refractive index of the sensing layer. Having multiple layers after metal layer, we can use effective refractive index $n_{\text {eff }}$ and can approximately express the sensing equation as [47] 
$\sin \theta_{S P R}=\frac{n_{e f f}}{n_{p}}$,

where $n_{\text {eff }}$ is the effective refractive index of the layers on the other side of the prism and $n_{p}$ is the refractive index of the prism material. $n_{\text {eff }}$ is defined as

$n_{\text {eff }}=\frac{2}{l_{d}} \int_{0}^{\infty} n(z) e^{-2 z / l_{d}} d z$

where $l_{d}$ is the penetration depth. Now in the case of our proposed structure with multiple dielectric layers on the other side of the metal, each layer thickness should be optimized so that minute change in the refractive index in the channel will create large variations in $n_{\text {eff }}$. This is a two dimensional optimization process where the goal is to make Rmin as low as possible while keeping the sensitivity as high as possible. For this purpose, each layer thickness is optimized one at a time keeping the thickness of all other layers fixed.Then we repeated the cycle multiple of time to get the global optimum values. Initially, we set the thickness of $\mathrm{Si}, \mathrm{BaTiO}_{3}$, Thiol layers at some arbitrary values and optimize $\mathrm{Ag}$ layer thickness to get minimum Rmin and maximum sensitivity. Then the same process is repeated for other layers as well based on sensitivity and Rmin. After that, we optimize the Ag thickness again with optimized thickness of $\mathrm{Si}, \mathrm{BaTiO}_{3}$ and thiol thickness. This optimization process is continued in an iterative manner until we get the most optimized structure possible. In Fig. 3(a), we show dependence of Rmin and sensitivity of the sensor against the thickness variation of Ag. Rmin gradually decreases with increasing thickness up to a certain thickness and then it starts increasing. Similar trend holds true for sensitivity as well but in opposite direction. We see that optimum thicknesses for minimum Rmin and maximum sensitivity are very close. Based on the obtained results, we select $50 \mathrm{~nm}$ as $\mathrm{Ag}$ thickness for our structure. Notably, our numerically optimized metal thickness matches well with theoretically obtained optimized value for metal layer as described in [48]. Figure 3(b), (c) and (d) shows the effect of $\mathrm{Si}, \mathrm{BaTiO}_{3}$, and thiol layer thickness variations on Rmin and sensitivity, respectively. For $\mathrm{Si}$, the variation pattern follows more or less same fashion as Ag. After $8 \mathrm{~nm}$ there is a drastic increase in Rmin. So, we choose $8 \mathrm{~nm}$ for $\mathrm{Si}$ thickness which gives maximum sensitivity with minimal Rmin. For $\mathrm{BaTiO}_{3}$, we select $11 \mathrm{~nm}$ as thickness for the same reason. Thiol optimization curve is quite different. Rmin was very low and fairly constant until $3 \mathrm{~nm}$ of thiol thickness, after which it starts increasing exponentially. However, the sensitivity peaks just before $4 \mathrm{~nm}$. As a result, we opt for 4 $\mathrm{nm}$ as thiol thickness to attain the best compromise between low Rmin and high sensitivity in our proposed structure. In this thickness the refractive index is 1.4 [36].

\section{Results and Discussion}

As mentioned in previous sections, the structure presented in this work consists of six layers. The sixth and final layer is the sensing layer, the refractive index $\left(n_{s}\right)$ of which will vary based on the sample attachment. These variations lead to the change in the resonance angle of SPR curve. From such variations, we obtain the sensitivity of the sensor according to Eqn. (8). Though sensitivity is the characteristic parameter for SPR biosensor's performance, other parameters such as Rmin, FWHM etc. have significant effect on the overall detection accuracy. Here, we will analyze our design based on different performance parameters and compare with other novel structures. We will also study the robustness of our sensor with different ligand-ligate pairs.

\section{Performance Analysis}

The performance of a sensor is determined by sensing accuracy as well as resolution. For SPR biosensor, resolution depends on FWHM of the reflectivity curve. The narrower the curve and hence lower FWHM, the better the resolution is. On the other hand, sensing accuracy depends on Rmin and sensitivity of the device. In Fig. 4(a) we show the SPR curve for two different refractive index of the sensing layer. We see that for a slight increase of refractive index, there is a noticeable variation in the resonance angle. This gives a sensitivity as high as $130.3 \mathrm{deg} / \mathrm{RIU}$ which is 7.6 times higher than the conventional Kretschmann configuration. In Fig. 4(b) and Fig. 4(c), we plot the coupled power and coupled fields versus incident angle, respectively. We see that both the coupled electric and magnetic field are maximum at the resonance angle. Same thing happens to the couple power as well which confirms the SPR occurrence at that particular incident angle. This clearly indicates the creation of evanescent wave at this specific angle and consequently we get a sharp dip in resonance curve. When light is incident at the resonance angle, maximum power transfer occurs from the source to the evanescent wave. Electric and

magnetic field profiles are shown in Fig. 4(d) along the direction of penetration of evanescent wave inside the sensing layer. Both the $\mathrm{E}$ - and $\mathrm{H}$-fields follow almost the same pattern. Field intensity peaks sharply at the vicinity of metal-dielectric interface and penetrates through the dielectric layers. As it passes through the dielectric layers, it dies out exponentially. Essentially, field strength is very strong within a very small distance where the analyte bonds with the ligand. This indicates a very narrow range of detectable distance. In our case, analyte bonding starts after $23 \mathrm{~nm}$ from the interface. We see that fields are very strong in this region which provides the enhanced sensitivity. 


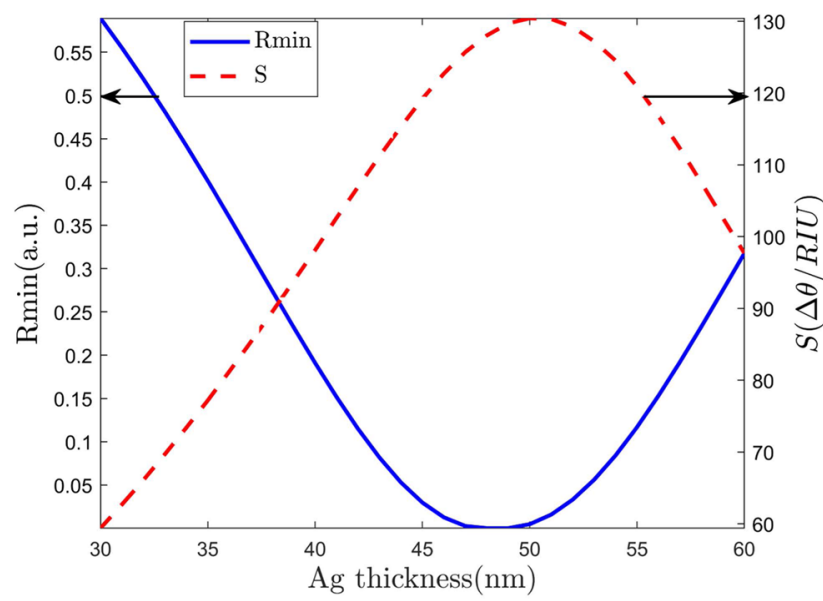

(a) Ag thickness Variation

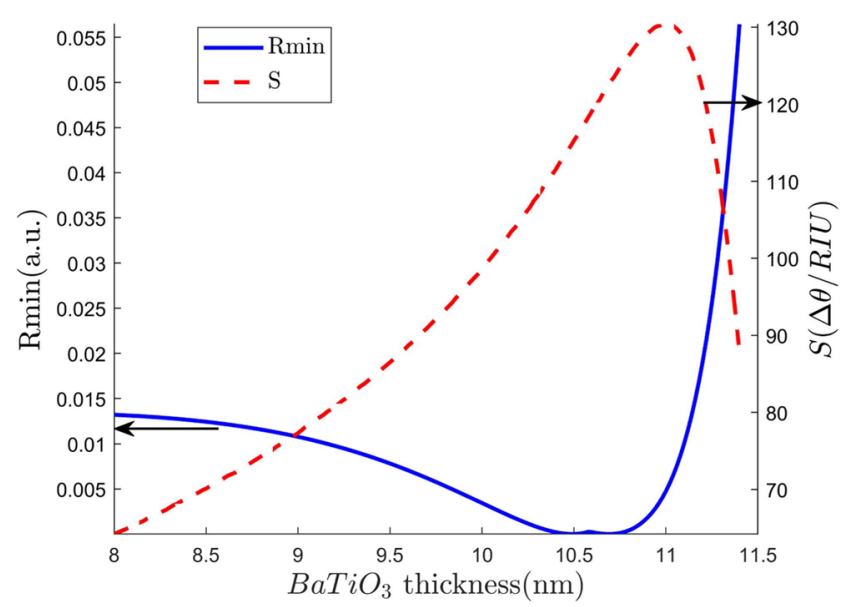

(c) $\mathrm{BaTiO}_{3}$ thickness Variation

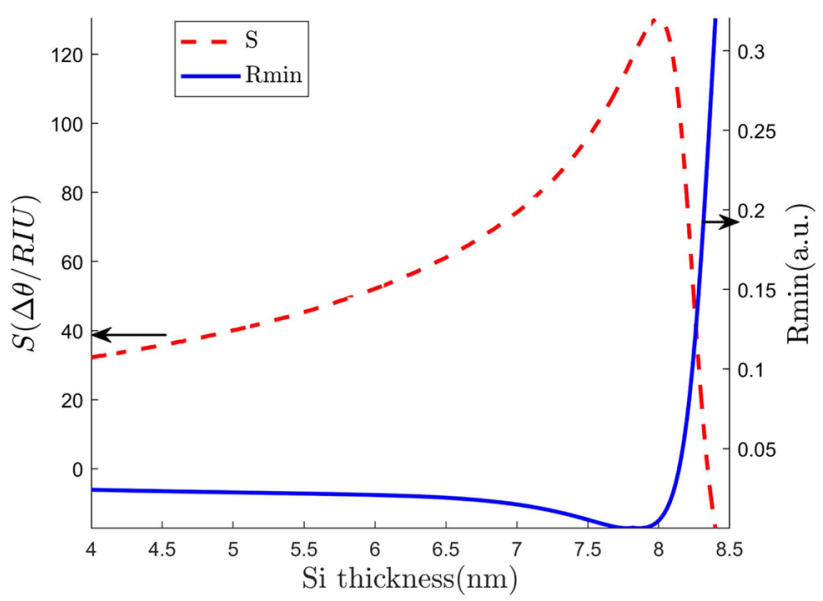

(b) Si thickness Variation

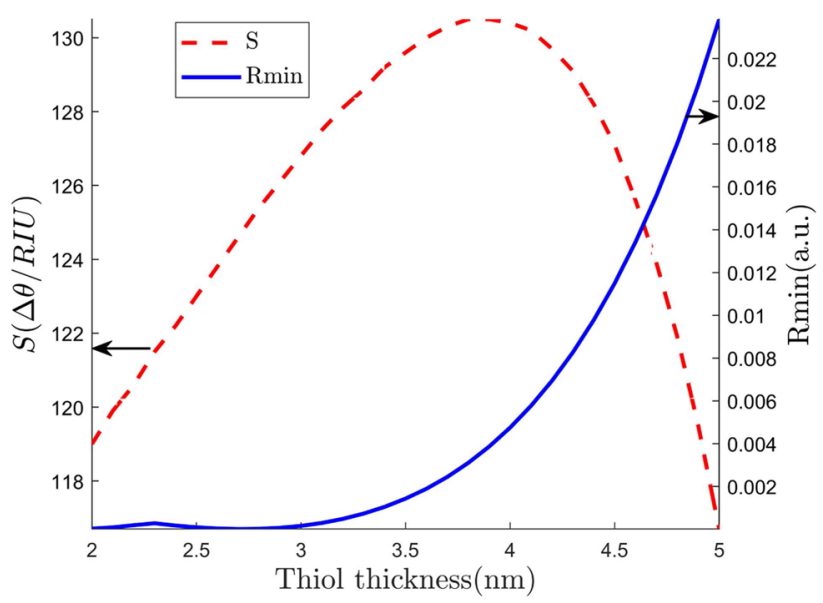

(d) Thiol tethered DNA thickness Variation

Fig. 3 Effect of different layer thickness on Rmin and sensitivity

We analyze all the structures from the basic Kretschmann configuration to our proposed design through subsequent intermediate structures. Gradual development of performance is noticed as we move from basic to proposed design. We vary refractive index from 1.33 to 1.34 for the sensing layer and observe the corresponding change in the

resonance angle. As we see in Fig. 5, sensitivity is significantly increased for the proposed structure. For the basic structure, we get the sensitivity to be only 18.4 degree/RIU. As we need a suitable receptor for Covid-19 sample, we put thiol-tethered DNA on top of Ag layer which increases sensitivity a little bit to 26.7 degree/RIU.

Inclusion of $\mathrm{Si}$ layer in between $\mathrm{Ag}$ and thiol-tethered DNA increases the sensitivity almost 3 times to 75.2 degree/ RIU. Finally for our proposed structure, we find sensitivity almost doubled to 130.3 degree/RIU. It is about 7.6 times compared to the basic configuration. This analysis is summarized in Table 1. In Fig. 6, we plot field strength against depth from the $\mathrm{Ag}-\mathrm{Si}$ interface along the direction of evanescent wave penetration (z-axis in our case). We see that the peak E-field undergoes a slight right shift with added layers. The rightmost red curve is our proposed structure. As we have seen earlier, our proposed structure contains some extra layers which shift the molecular binding region toward right further. With the peak E-field shifted to the right, molecules now bond in the significant electric field region. This happens for all intermediate structures.

We also investigate our structure using an FDTD solver to verify our findings. As shown in Fig. 7, we get similar response for our proposed structure from the FDTD method as we get in transfer matrix method. This confirms robustness of our investigations.

Though our main goal is to maximize the performance in angular interrogation method, our structure shows promising 


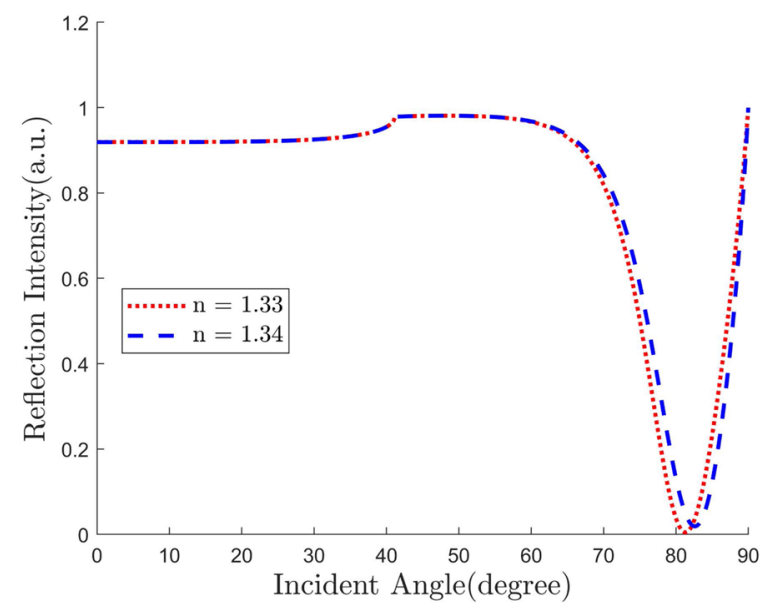

(a) Reflected light intensity versus source incident angle

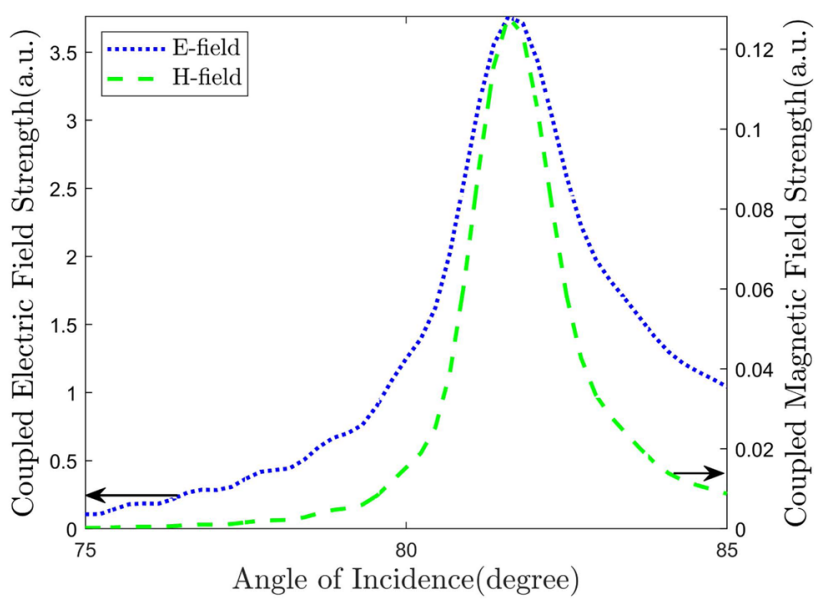

(c) Coupled field strength versus source incident angle

Fig. 4 Performance analysis of the proposed structure

results in wavelength interrogation process also. To investigate the proposed structure in wavelength interrogation method, we fixed the incident angle at 81 degree. Then we measure the reflected light at different wavelength and observe the reflectance dip. We vary the refractive index of the sensing layer from 1.33 to 1.34 and observe the corresponding shift in resonance wavelength. We notice a significant red shift in the SPR curve as shown in Fig. 8. We use the following equation to determine the sensitivity,

$S_{\lambda}=\frac{d \lambda}{d n_{s}}$,

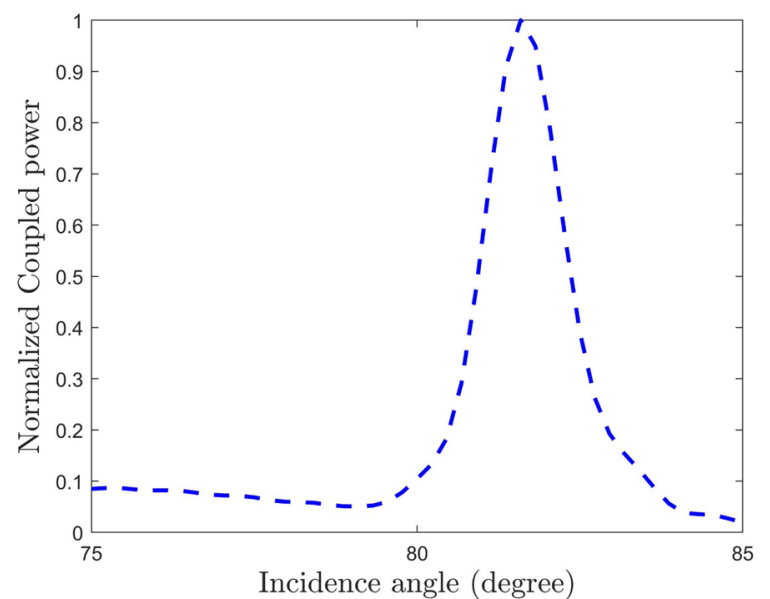

(b) Normalised coupled power versus source incident angle

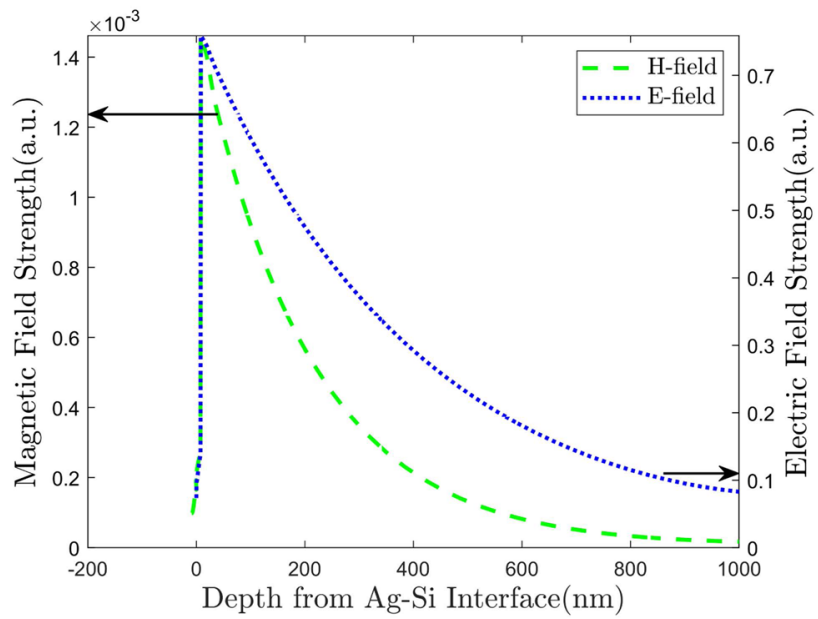

(d) Optical near field distribution of the plasmonic films

where $d \lambda$ is the shift of resonance wavelength due to the change of refractive index of the sensing layer, $d n_{s}$. We get the sensitivity as high as $300 \mathrm{~nm} / \mathrm{RIU}$.

We now investigate the sensing performance of our structure for SARS-COV-2 detection. We use thiol-tethered DNA as a ligand layer as it has been proved to be a good receptor of SARS-COV-2. We collect data related to SARS-COV-2 from literature [49]. In the proposed sensing scheme, samples collected from human nasopharyngeal swabs are passed through the sensing channel in a liquid solution. When hybridization happens between SARS-CoV-2 RNA (RdRpCOVID sequence) from sample with thiol-tethered DNA of 
Table 1 Different Layers' Effect on Sensitivity

\begin{tabular}{lc}
\hline Sensor Configuration & $\begin{array}{l}\text { Sensitivity } \\
\text { (degree/RIU) }\end{array}$ \\
\hline Silver & 18.4 \\
Silver+Silicon & 26.7 \\
Silver+Silicon+ & 71.9 \\
Basic+Silicon+Thiol & 75.2 \\
Proposed Architecture & 130.4 \\
\hline
\end{tabular}

receptor molecules, it causes a significant SPR angle shift (as high as 12 degree) as demonstrated in Fig. 9. Moreover, the reflectance dip is accompanied with very low Rmin (0.01587) and FWHM (11.86), which shows the prowess of

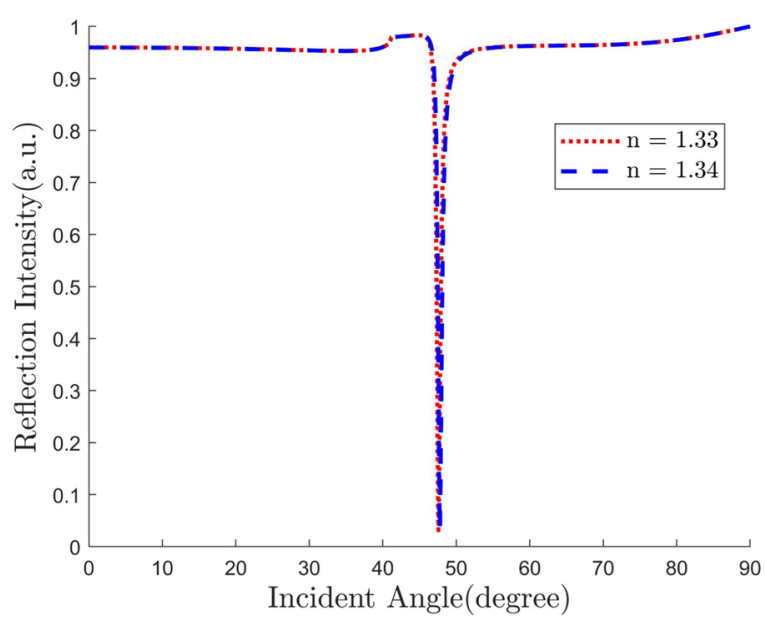

(a) Basic configuration

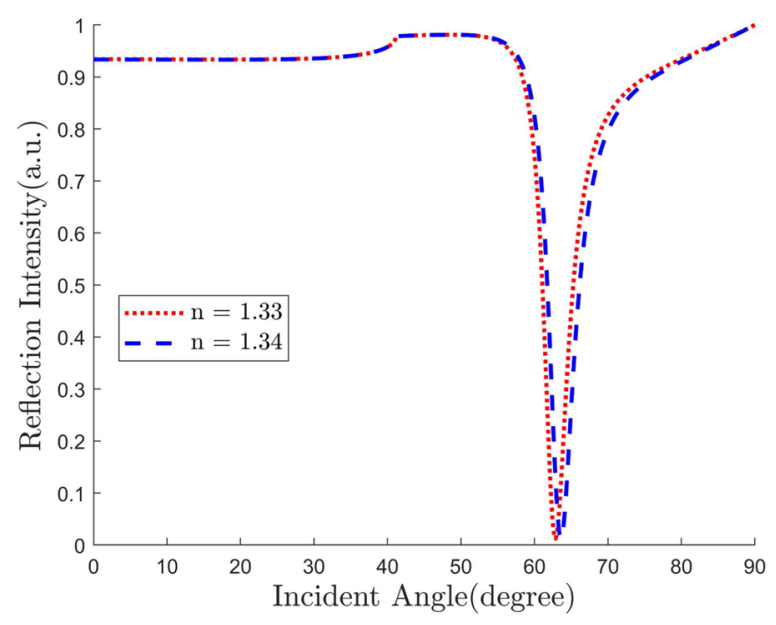

(c) Ag-Si-Thiol tethered DNA our design as a potential plasmonic sensor for highly sensitive SARS-COV-2 detection.

\section{Comparison with Other Reported Structures}

Finally, we compare the performance parameters of our design with existing structures in literature. The performance metrics taken into account for comparison purposes are sensitivity, Rmin and FWHM. While sensitivity is the most important performance parameter under consideration, other two are also crucial for sensing accuracy. Hence, we define FOM according to Eqn. (7) as a benchmark for comparison. We show the findings in Table 2. From the table, we see that the proposed design shows higher sensitivity than majority of structures. While some designs have

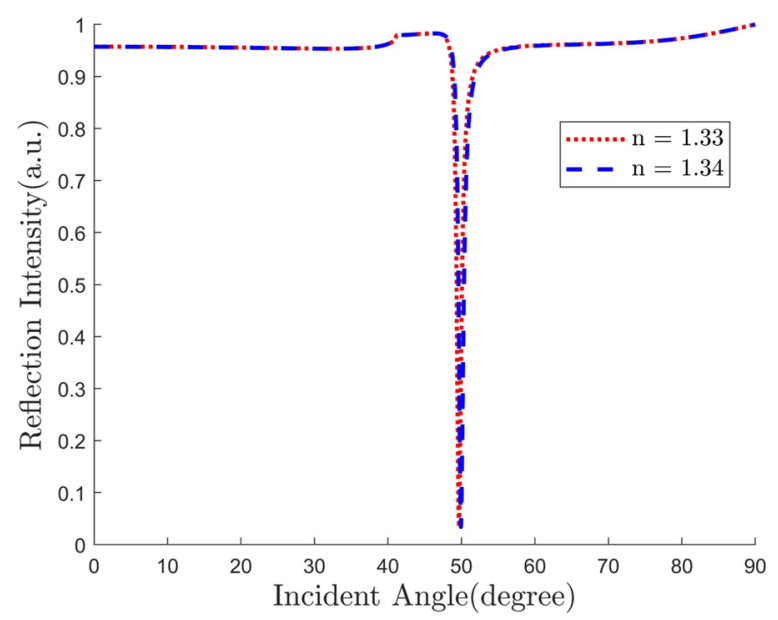

(b) Basic configuration with Thiol tethered DNA

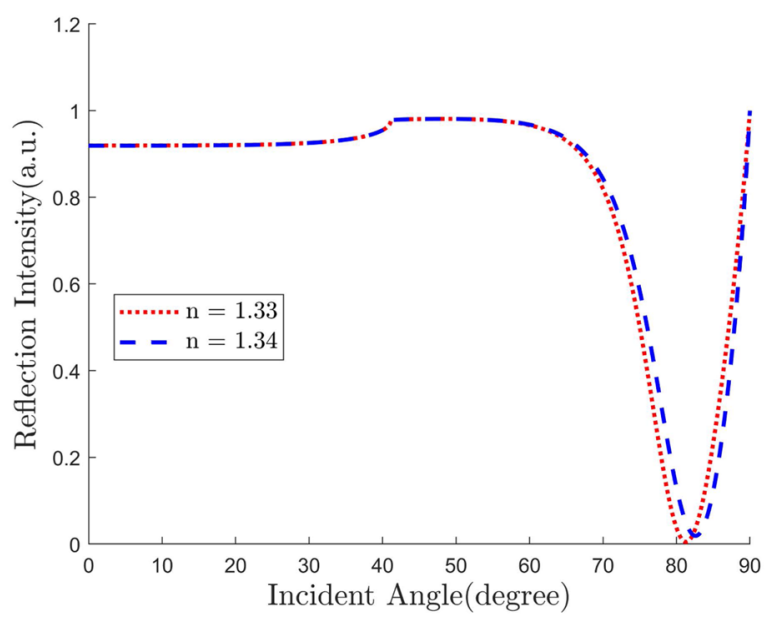

(d) Proposed Structure

Fig. 5 Reflectance versus incident angle 


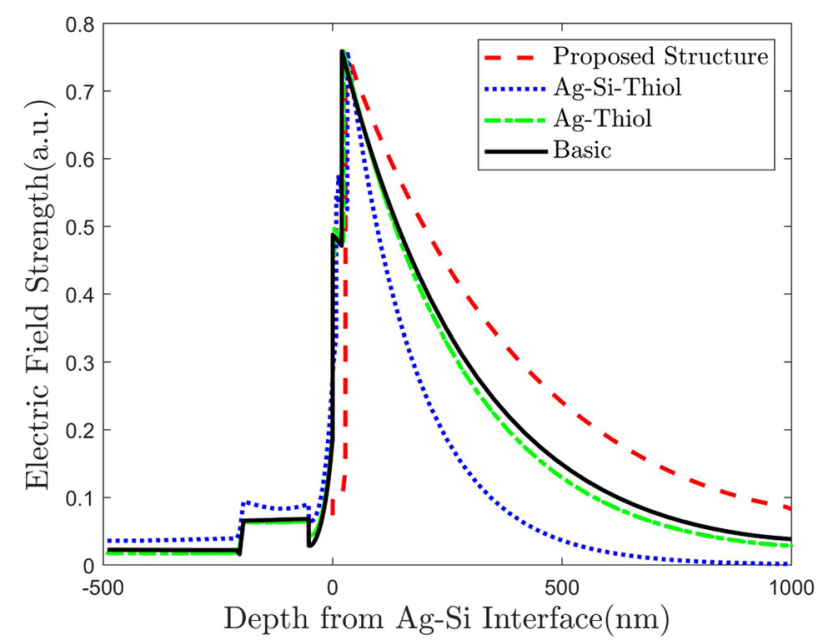

Fig. 6 Evolution of optical near field distribution of the plasmonic films for different structures

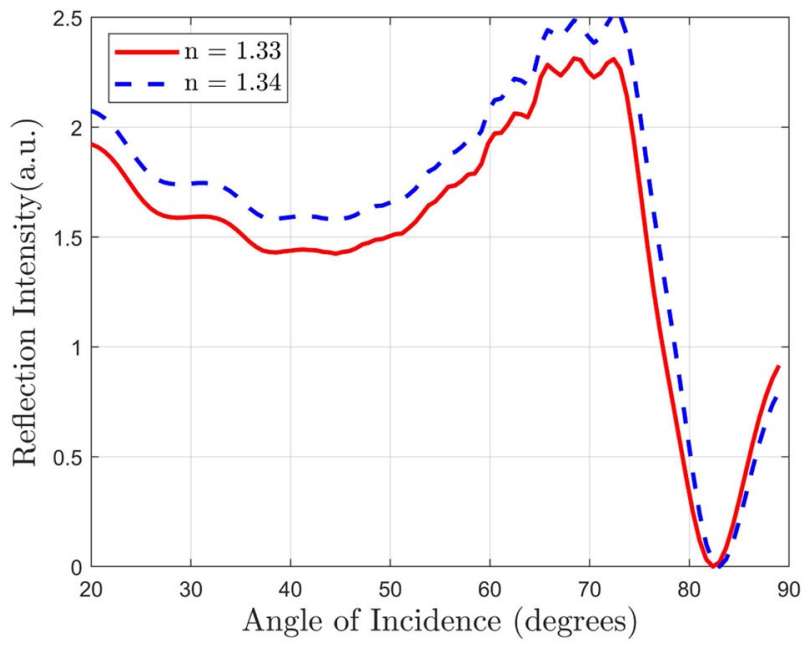

Fig. 7 Reflected light intensity versus source incident angle

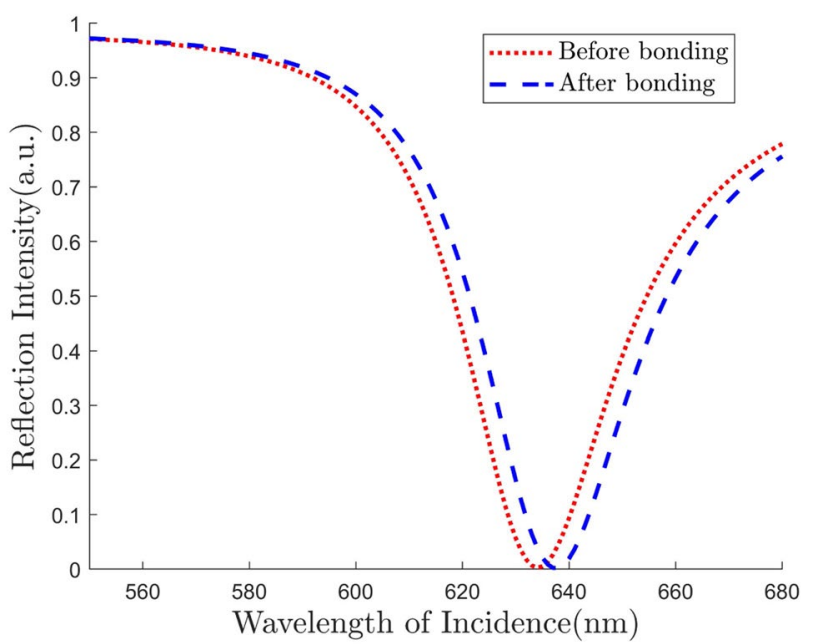

Fig. 8 Reflectance curve for wavelength interrogation of the proposed structure

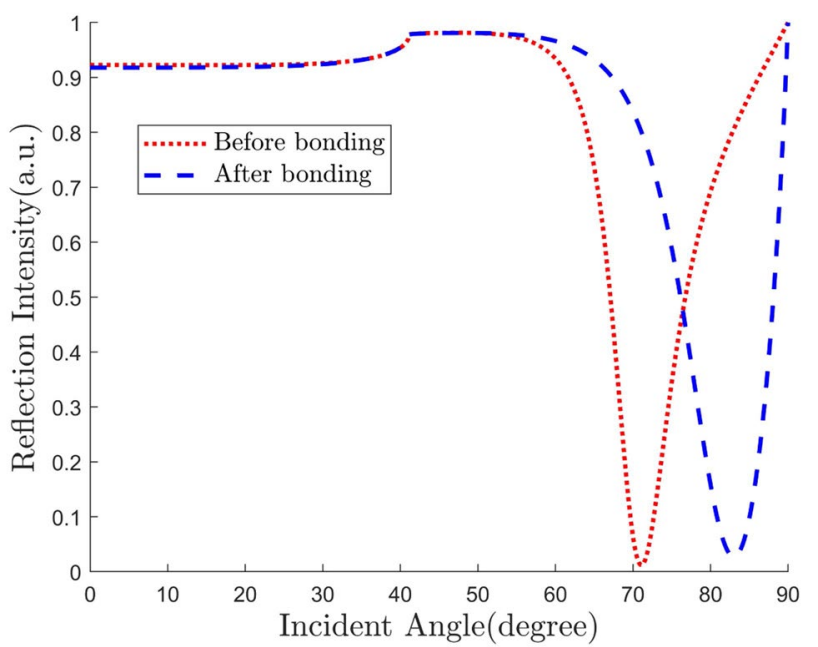

Fig. 9 Reflectance curve for detection of SARS-COV-2
Table 2 Sensing performance comparison with other published results

\begin{tabular}{|c|c|c|c|c|}
\hline Sensor configuration & Sensitivity & FWHM & Rmin & FOM \\
\hline Proposed structure & 130.3 & 11.86 & 0.01587 & 692.28 \\
\hline $\mathrm{Au}-\mathrm{Si}-\mathrm{MX}_{2}[45]$ & 147.88 & 16.2417 & 0.024099 & 377.81 \\
\hline $\mathrm{TiO}_{2}-\mathrm{SiO}_{2}-\mathrm{Ag}-\mathrm{MoS}_{2}$-Graphene [50] & 98 & 1.102 & Not reported & Not reported \\
\hline $\mathrm{Au}-\mathrm{MoS}_{2}$-Graphene $[51]$ & 89.29 & 6.80 & 0.025 & 525.2 \\
\hline Cr-Ag-ITO [52] & 68.77 & 1.64 & 0.0903 & 464.37 \\
\hline ZnO-Ag-Au-graphene [53] & 76 & 5 & 0.04 & 380 \\
\hline $\mathrm{ZnO}-\mathrm{Au}-\mathrm{MoS}_{2}$-Graphene [54] & 101.58 & 6.73 & Not reported & Not reported \\
\hline $\mathrm{Cu}-\mathrm{MoS}_{2}$-Graphene [55] & 79.12 & 2.89 & 0.0785 & 348.755 \\
\hline Air- $\mathrm{MoS}_{2}$-Al-MoS -Graphene [56] & 190.83 & 19 & 0.04 & 251.09 \\
\hline Mirrored bilayer of Au- $\mathrm{MoS}_{2}$-Graphene [47] & 75.2 & 17 & 0.1 & 44.23 \\
\hline Au-Graphene [57] & 53.71 & 5 & 0.1252 & 85.79 \\
\hline Chalcogenide Prism-Au-Graphene [58] & 40 & 2.5 & 0.1 & 160 \\
\hline Graphene nanoribbons [59] & 160 & 0.86 & not reported & not reported \\
\hline
\end{tabular}


Table 3 Robustness

\begin{tabular}{ll}
\hline Ligand-Ligate Pair & Sensitivity \\
\hline BSA-Phospholipid & 121.6 \\
BSA-Egg Yolk & 121.46 \\
\hline
\end{tabular}

obtained better sensitivity compared to ours, they simultaneously suffer from higher Rmin and FWHM, and hence lower FOM. In terms of FOM, clearly our proposed structure outperforms other competitive counterparts. Moreover, For immobilization of SARS COV-2 virus, we have to grow thiol-tethered DNA layer which reduce general sensitivity of our structure. None of these structures is studied with thiol-tethered DNA as a functionalizing layer.

\section{Robustness Analysis}

For a sensor architecture to be successful in practice, ideally it should be able to provide consistent performance over a wide range of sensing molecule configurations. That is why, we also studied and analyzed our proposed framework for other ligand-ligate pairs. Notably, we observe similar improvement in performance for different receptor and sensing samples which demonstrates the robustness of our structure. These results are given in Table 3 .

\section{Conclusion}

We have analyzed an SPR based novel biosensor for labelfree and real-time detection of SARS-COV-2. Extensive numerical simulations have been carried out for structural optimization and performance analysis. Transfer matrix method as well as FDTD technique have been employed to perform the simulations. Our results have shown promising indications for the usage of the proposed sensor configuration as a potential detector of SARS-COV-2. We have considered not only sensitivity but also other standard evaluation metrics such as FWHM, Rmin to demonstrate the performance of our design. Furthermore, we have defined a FOM to encapsulate all the performance parameters in a single evaluation quantity and compared with other existing structures. This investigation has demonstrated competitive effectiveness of our proposed structure over other designs. Moreover, the robustness of our sensor has been verified with two other different ligand-ligate pairs. The study is limited in the sense that the results are primarily numerical and experimental validation of such enhancements are required which is out of scope here and focus of future work. However, for experimental investigations, a well-designed and numerically evaluated sensor architecture is crucial, therein lies the significance of the presented study.
Author Contributions Syed Mohammad Ashab Uddin has given the main idea and done the simulations. Ehsan Kabir helped to collect relevant information. Sayeed Shafayet Chowdhury provided the code and supervised the work. All the authors have contributed in writing the manuscript.

Funding Information The authors did not receive support from any organization for the submitted work.

Availability of Data and Materials All data generated or analyzed during this study are included in this published article [and its supplementary information files].

\section{Declarations}

Ethical Approval We confirm that this work doesn't have any human participation or clinical Data.

Consent to Participate We confirm that this work doesn't have any human participation or clinical Data.

Consent to Publish We confirm that this work doesn't have any human participation or clinical Data.

Conflict of Interest We have no conflicts of interest to disclose.

\section{References}

1. Bhalla N, Pan Y, Yang Z, Payam AF (2020) Opportunities and challenges for biosensors and nanoscale analytical tools for pandemics: Covid-19. ACS nano

2. Organization WH (2020) Coronavirus disease 2019 (covid-19): situation report-88. WHO

3. WHO Coronavirus disease (COVID-19) dashboard, Accessed: 2020-10-09. https://covid19.who.int/

4. Udugama B, Kadhiresan P, Kozlowski HN, Malekjahani A, Osborne M, Li VY, Chen H, Mubareka S, Gubbay JB, Chan WC (2020) Diagnosing covid-19: the disease and tools for detection. ACS Nano 14(4):3822-3835

5. Seo G, Lee G, Kim MJ, Baek SH, Choi M, Ku KB, Lee CS, Jun S, Park D, Kim HG et al (2020) Rapid detection of covid19 causative virus (sars-cov-2) in human nasopharyngeal swab specimens using field-effect transistor-based biosensor. ACS Nano 14(4):5135-5142

6. Chua F, Armstrong-James D, Desai SR, Barnett J, Kouranos V, Kon OM, José R, Vancheeswaran R, Loebinger MR, Wong $\mathrm{J}$ et al (2020) The role of ct in case ascertainment and management of covid-19 pneumonia in the uk: insights from highincidence regions. Lancet Respir Med 8(5):438-440

7. Abedin S, Kenison J, Vargas C, Potma EO (2019) Sensing biomolecular interactions by the luminescence of a planar gold film. Anal Chem 91(24):15883-15889

8. Almajhadi MA, Uddin SMA, Wickramasinghe HK (2019) Observation of nanoscale opto-mechanical molecular damping; origin of spectroscopic contrast in photo induced force microscopy. arXiv preprint arXiv: 1911.05190

9. Almajhadi MA, Uddin SMA, Wickramasinghe HK (2020) Observation of nanoscale opto-mechanical molecular damping; origin of spectroscopic contrast in infrared photo induced force microscopy. In CLEO: Science and Innovations, Optical Society of America, pp. SW3G-7 
10. Bijalwan A, Rastogi V (2018) Gold-aluminum-based surface plasmon resonance sensor with a high quality factor and figure of merit for the detection of hemoglobin. Appl Opt 57(31):9230-9237

11. Chowdhury SS, Hasan MS, Sharmin R (2019) Robust heart rate estimation from ppg signals with intense motion artifacts using cascade of adaptive filter and recurrent neural network. In TENCON 2019-2019 IEEE Region 10 Conference (TENCON). IEEE, pp. 1952-1957

12. Chowdhury SS, Hyder R, Hafiz MSB, Haque MA (2016) Realtime robust heart rate estimation from wrist-type ppg signals using multiple reference adaptive noise cancellation. IEEE $\mathbf{J}$ Biomed Health Info 22(2):450-459

13. Chowdhury SS, Hyder R, Shahanaz C, Fattah SA (2017) Robust single finger movement detection scheme for real time wheelchair control by physically challenged people. In 2017 IEEE Region 10 Humanitarian Technology Conference (R10-HTC). IEEE, pp. 773-777

14. Chowdhury SS, Lee C, Roy K (2020) Towards understanding the effect of leak in spiking neural networks. arXiv preprint arXiv:2006.08761

15. Fattah SA, Rahman NM, Maksud A, Foysal SI, Chowdhury RI, Chowdhury SS, Shahanaz C (2017) Stetho-phone: Lowcost digital stethoscope for remote personalized healthcare. In 2017 IEEE Global Humanitarian Technology Conference (GHTC). IEEE, pp. 1-7

16. Hyder R, Chowdhury SS, Fattah S (2016) A. Real-time nonintrusive eye-gaze tracking based wheelchair control for the physically challenged. In 2016 IEEE EMBS Conference on Biomedical Engineering and Sciences (IECBES). IEEE, pp. $784-787$

17. Kurihara K, Suzuki K (2002) Theoretical understanding of an absorption-based surface plasmon resonance sensor based on kretchmann's theory. Anal Chem 74(3):696-701

18. Maksud A, Chowdhury RI, Chowdhury TT, Fattah SA, Shahanaz C, Chowdhury SS (2017) Low-cost eeg based electric wheelchair with advanced control features. In TENCON 2017-2017 IEEE Region 10 Conference. IEEE, pp. 2648-2653

19. Shahnaz C, Maksud A, Fattah SA, Chowdhury SS (2017) Lowcost smart electric wheelchair with destination mapping and intelligent control features. In 2017 IEEE International Symposium on Technology and Society (ISTAS). IEEE, pp. 1-6

20. Shahnaz C, Sampad MJN, Adhikary D, Uchayash SM, Mahdia M, Chowdhury SS, Fattah SA (2017) Smart-hat: Safe and smooth walking assistant for elderly people. In 2017 IEEE International Symposium on Technology and Society (ISTAS). IEEE, pp. 1-7

21. Otto A (1968) Excitation of nonradiative surface plasma waves in silver by the method of frustrated total reflection. Zeitschrift für Physik A Hadrons and nuclei 216(4):398-410

22. Kretschmann E, Raether H (1968) Radiative decay of nonradiative surface plasmons excited by light. Z. Naturforsch. a 23(12):2135-2136

23. Chowdhury SS, Uddin SMA, Kabir E, Chowdhury AM (2017) Detection of dna mutation, urinary diseases and blood diseases using surface plasmon resonance biosensors based on kretschmann configuration. In 2017 International Conference on Electrical, Computer and Communication Engineering (ECCE). IEEE, pp. 662-665

24. Qiu G, Gai Z, Tao Y, Schmitt J, Kullak-Ublick GA, Wang J (2020) Dual-functional plasmonic photothermal biosensors for highly accurate severe acute respiratory syndrome coronavirus 2 detection. ACS Nano 14(5):5268-5277

25. Rahman MS, Hasan MR, Rikta KA, Anower M (2018) A novel graphene coated surface plasmon resonance biosensor with tungsten disulfide (ws2) for sensing dna hybridization. Opt Mat 75:567-573
26. Verma A, Prakash A, Tripathi R (2015) Sensitivity enhancement of surface plasmon resonance biosensor using graphene and air gap. Opt Com 357:106-112

27. Bhatia P, Gupta BD (2011) Surface-plasmon-resonance-based fiber-optic refractive index sensor: sensitivity enhancement. Appl Opt 50(14):2032-2036

28. Lahav A, Auslender M, Abdulhalim I (2008) Sensitivity enhancement of guided-wave surface-plasmon resonance sensors. Opt Lett 33(21):2539-2541

29. Verma R, Gupta BD, Jha R (2011) Sensitivity enhancement of a surface plasmon resonance based biomolecules sensor using graphene and silicon layers. Sens Act B: Chem 160(1):623-631

30. Wang Q, Niu LY, Jing JY, Zhao WM (2020) Barium titanate film based fiber optic surface plasmon sensor with high sensitivity. Opt Laser Tech 124:105899

31. Shang J, Ye G, Shi K, Wan Y, Luo C, Aihara H, Geng Q, Auerbach A, Li F (2020) Structural basis of receptor recognition by sarscov-2. Nature 581(7807):221-224

32. Dai X, Liang Y, Zhao Y, Gan S, Jia Y, Xiang Y (2019) Sensitivity enhancement of a surface plasmon resonance with tin selenide (snse) allotropes. Sensors 19(1):173

33. Liu L, Wang M, Jiao L, Wu T, Xia F, Liu M, Kong W, Dong L, Yun M (2019) Sensitivity enhancement of a graphene-barium titanate-based surface plasmon resonance biosensor with an ag-au bimetallic structure in the visible region. JOSA B 36(4):1108-1116

34. Lin Z, Jiang L, Wu L, Guo J, Dai X, Xiang Y, Fan D (2016) Tuning and sensitivity enhancement of surface plasmon resonance biosensor with graphene covered au-mos 2-au films. IEEE Photonics J 8(6): 1-8

35. Palik ED (1998) Handbook of optical constants of solids, vol. 3. Academic press

36. Peterlinz KA, Georgiadis RM, Herne TM, Tarlov MJ (1997) Observation of hybridization and dehybridization of thiol-tethered dna using two-color surface plasmon resonance spectroscopy. J Am Chem Soc 119(14):3401-3402

37. Asghari A, Wang C, Yoo KM, Dalir H, Chen RT (2020) Fast accurate point of care covid-19 pandemic diagnosis enabled through advanced lab-on-a-chip optical biosensors: Opportunities and challenges. arXiv preprint arXiv:2008.08572

38. Zhang Q, Zhong L, Tang P, Yuan Y, Liu S, Tian J, Lu X (2017) Quantitative refractive index distribution of single cell by combining phase-shifting interferometry and afm imaging. Sci Rep 7(1): $1-10$

39. Van Manen HJ, Verkuijlen P, Wittendorp P, Subramaniam V, Van den Berg TK, Roos D, Otto C (2008) Refractive index sensing of green fluorescent proteins in living cells using fluorescence lifetime imaging microscopy. Biophys J 94(8):L67-L69

40. Wölfel R, Corman VM, Guggemos W, Seilmaier M, Zange S, Müller MA, Niemeyer D, Jones TC, Vollmar P, Rothe C et al (2020) Virological assessment of hospitalized patients with covid2019. Nature 581(7809):465-469

41. Schasfoort RB (2017) Introduction to surface plasmon resonance. Royal Society of Chemistry

42. Anower MS, Rahman MS, Rikta KA (2018) Performance enhancement of graphene-coated surface plasmon resonance biosensor using tungsten disulfide. Opt Eng 57(1):017114

43. Yamamoto M (2002) Surface plasmon resonance (spr) theory: tutorial. Rev Polarograph 48(3):209-237

44. Mohammed ZH (2019) The fresnel coefficient of thin film multilayer using transfer matrix method tmm. In IOP Conference Series: Materials Science and Engineering. vol. 518, IOP Publishing, p. 032026

45. Ouyang Q, Zeng S, Jiang L, Hong L, Xu G, Dinh X-Q, Qian J, He $S$, Qu J, Coquet P et al (2016) Sensitivity enhancement of transition metal dichalcogenides/silicon nanostructure-based surface plasmon resonance biosensor. Sci Rep 6:28190 
46. Kabir E, Uddin SMA, Chowdhury SS (2020) Optimization of surface plasmon resonance biosensor for analysis of lipid molecules. arXiv preprint arXiv:2007.08607

47. Chowdhury SS, Uddin SMA, Kabir E (2020) Numerical analysis of sensitivity enhancement of surface plasmon resonance biosensors using a mirrored bilayer structure. Photon Nanostruct-Fund Appl. 100815

48. Uddin SMA, Chowdhury SS, Kabir E (2017) A theoretical model for determination of optimum metal thickness in kretschmann configuration based surface plasmon resonance biosensors. In 2017 International Conference on Electrical, Computer and Communication Engineering (ECCE). IEEE, pp. 651-654

49. Wang Q, Wu J, Wang H, Gao Y, Liu Q, Mu A, Ji W, Yan L, Zhu Y, Zhu C, et al (2020) Structural basis for rna replication by the sars-cov-2 polymerase. Cell

50. Moznuzzaman M, Islam MR, Hossain MB, Mehedi IM (2020) Modeling of highly improved spr sensor for formalin detection. Res Phys 16:102874

51. Rahman MS, Anower MS, Hasan MR, Hossain MB, Haque MI (2017) Design and numerical analysis of highly sensitive aumos2-graphene based hybrid surface plasmon resonance biosensor. Opt Com 396:36-43

52. Gan S, Menon P, Mohamad N, Jamil N, Majlis B (2019) Fdtd simulation of kretschmann based cr-ag-ito spr for refractive index sensor. Materials Today: Proceedings 7:668-674
53. Kumar R, Kushwaha AS, Srivastava M, Mishra H, Srivastava S (2018) Enhancement in sensitivity of graphene-based zinc oxide assisted bimetallic surface plasmon resonance (spr) biosensor. Appl Phys A 124(3):235

54. Kushwaha AS, Kumar A, Kumar R, Srivastava S (2018) A study of surface plasmon resonance (spr) based biosensor with improved sensitivity. Photon Nanostruct-Fund Appl 31:99-106

55. Maurya J, Prajapati Y (2016) A comparative study of different metal and prism in the surface plasmon resonance biosensor having mos 2-graphene. Opt Quan Electron 48(5):280

56. Wu L, Jia Y, Jiang L, Guo J, Dai X, Xiang Y, Fan D (2016) Sensitivity improved spr biosensor based on the mos2/graphene-aluminum hybrid structure. J Lightwave Tech 35(1):82-87

57. Rahman MS, Anower MS, Bashar LB, Rikta KA (2017) Sensitivity analysis of graphene coated surface plasmon resonance biosensors for biosensing applications. Sens Bio-sens Res 16:41-45

58. Verma A, Prakash A, Tripathi R (2016) Sensitivity improvement of graphene based surface plasmon resonance biosensors with chaclogenide prism. Optik 127(4):1787-1791

59. Bijalwan A, Singh BK, Rastogi V (2020) Surface plasmon resonance-based sensors using nano-ribbons of graphene and wse 2. Plasmonics 1-9

Publisher's Note Springer Nature remains neutral with regard to jurisdictional claims in published maps and institutional affiliations. 